\title{
Will the inflation genie stay in the bottle?
}

Written by: Dan Andrews, OECD Economics Department

Last update: 3 March 2020

While the evidence shows that global integration has pushed down inflation, a stalling in globalisation could cause that trend to slip into reverse. Here is why.

Dan andrews, peter gal and william witheridge Could there be a sustained pick-up in inflation under way in the US after years of subdued price pressures, as market commentators have been speculating? Along with continued solid US jobs growth and low unemployment, there are tentative signs of higher wage growth, while fiscal stimulus has also been boosting growth for some time. Global growth has also been strong in recent years.

Will the inflation genie stay in the bottle? Chart 1

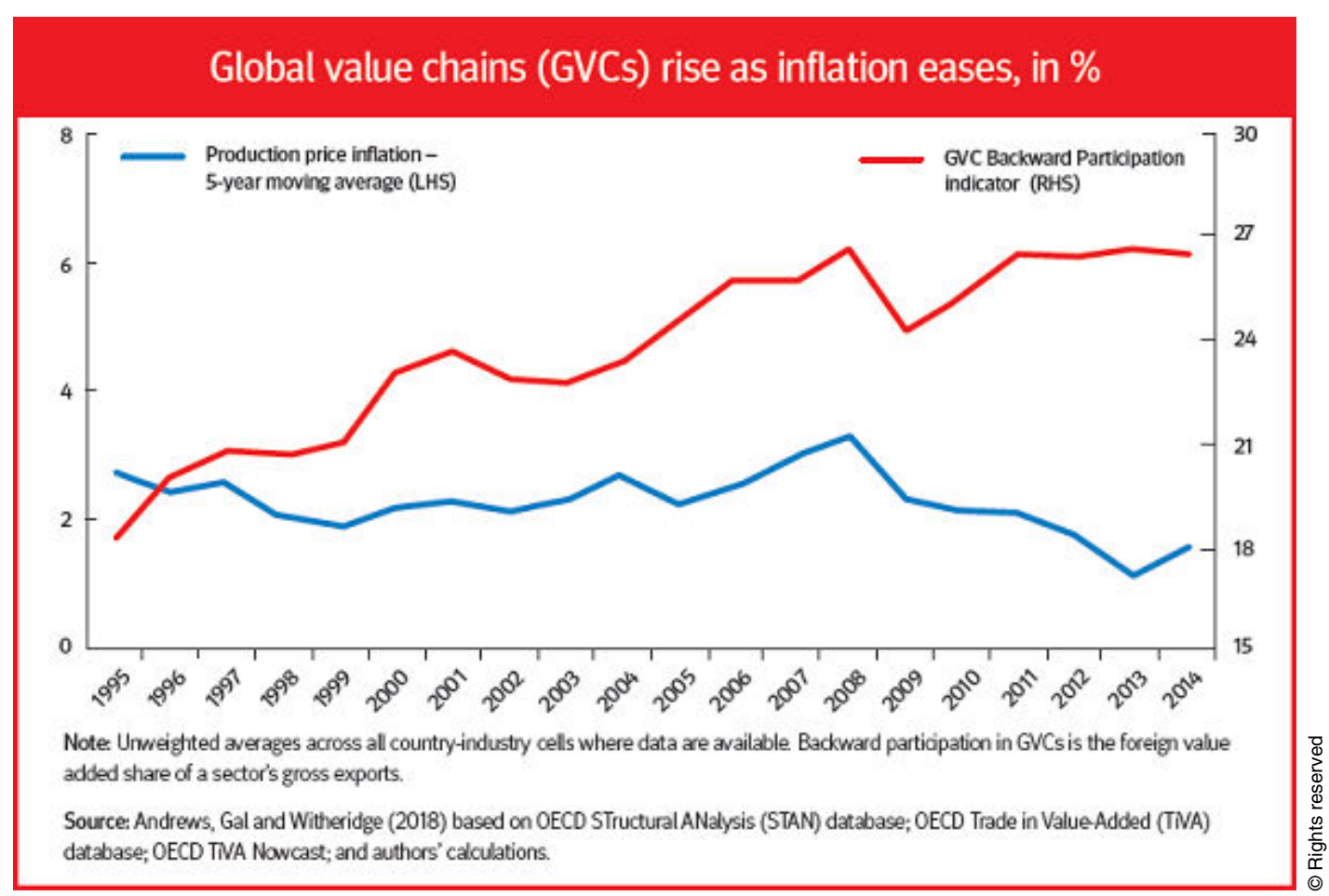

Chart 1 for Will the inflation genie stay in the bottle? GVCs rise as inflation eases, in \%

Our new OECD working paper (see references) identifies a robust negative relationship between inflation and globalisation, and offers some novel insights. In particular, globalisation appears to have stalled since the crisis, especially with 
mounting tensions around trade policy. There is also mounting evidence of rising market power in services sectors. Together, these trends risk letting the inflation genie out of the bottle.

\section{Chart 2 for Will the inflation genie stay in the bottle?}

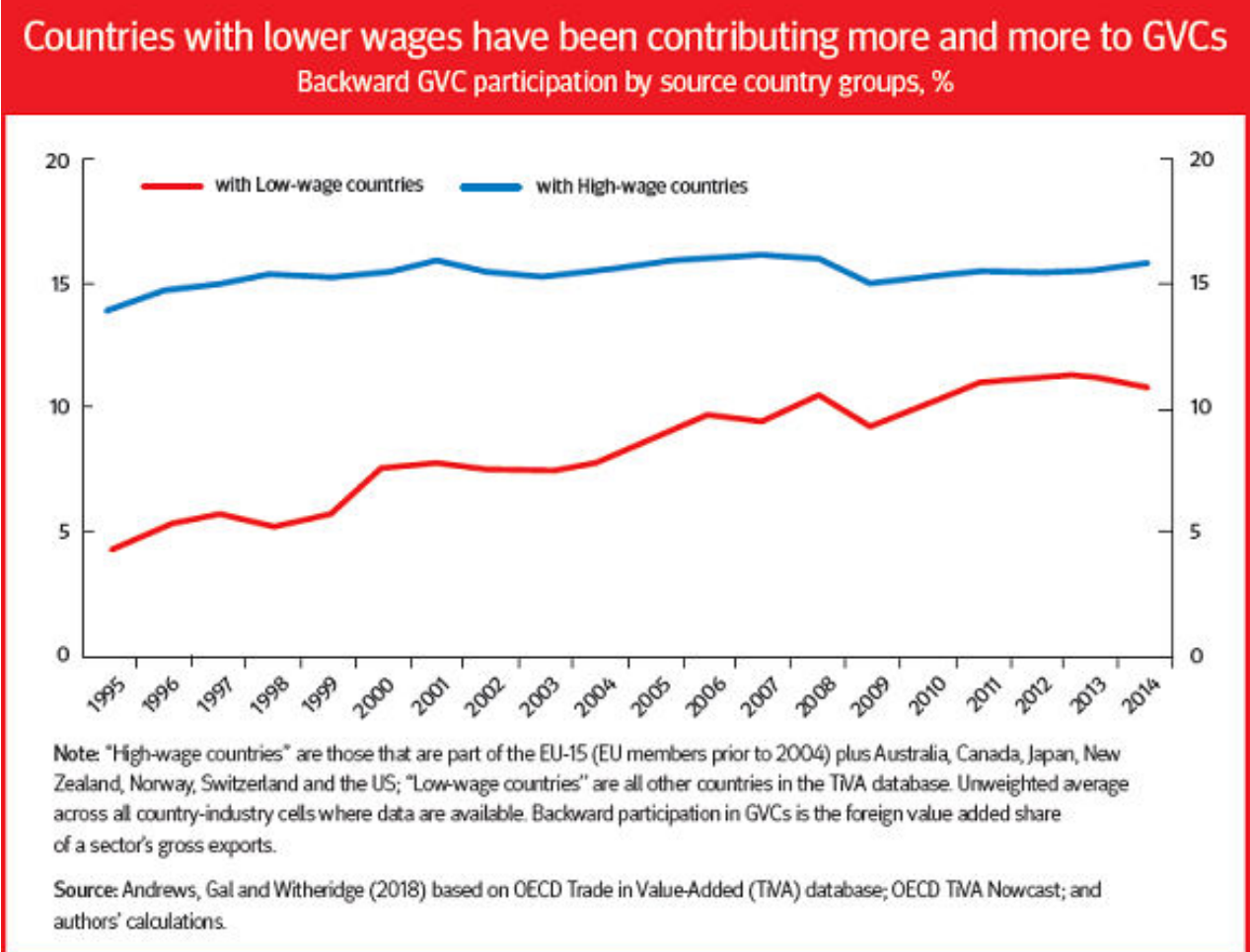

Chart 2 for Will the inflation genie stay in the bottle? Countries with lower wages have been contributing more and more to GVCs

How important is globalisation for domestic inflation? Some have argued that rising integration along global value chains (GVCs) has accentuated the importance of global factors-particularly slack in world demand-for domestic inflation, while other recent research disputes this conjecture. GVC integration expanded significantly from 1995 until the crisis, while inflation remained relatively subdued. In the post-crisis period, GVCs flattened off and remained around the pre-crisis peak while producer price inflation fell dramatically and remained very low on average across industries in OECD countries (see chart).

Our analysis using cross-country OECD data on prices and global value chains by industry (rather than at the country level) shows that stronger backward GVC participation-that is, domestic producers relying more on foreign value-added content-is associated with lower producer price inflation at industry level. For example, we estimate that the rise in GVCs from the mid-1990s up to the crisis reduced annual producer price inflation by 0.15 percentage points on average, with this effect more than double in some OECD countries. 
Our analysis also reveals that greater reliance by domestic producers on foreign value-added content is associated with lower wages and rising productivity in the importing countries and industries, especially when low-wage countries become an integral part of their supply chains. This has likely contributed to lower inflation in recent years as the composition of source countries in GVCs has increasingly been low-wage ones, as our second chart shows. Therefore, inflation in advanced economies can remain low if the composition of GVCs continues to shift towards low-wage countries.

chart

Moreover, a high level of GVC integration can also dampen producer price inflation by accentuating the impact of global economic slack on domestic inflation. This implies that weak global demand has a larger downward impact on domestic inflation when GVC participation is higher. For example, countries operating below potential, with an average global output gap of $-1.5 \%$ in 2014 . had annual producer price inflation that was on average 0.25 percentage points lower in 2014 than for 1996 GVC levels. This inflation figure drops more than 0.5 percentage points lower, however, for those countries that experienced a particularly large rise in GVC participation. But with slowing expansion of global value chains since the crisis, coupled with stronger aggregate demand and output gaps closing or in positive territory in many countries, this could lead to greater inflationary pressures in the medium term.

\section{Competition problem}

The third longer-term trend posing an upside risk to inflation is declining competition and market contestability. Cross-country firm-level data show an increasing trend in mark-ups, which suggests rising market power in services sectors. This upward trend in mark-ups is consistent with other estimates for the US and other OECD countries. If market power continues to rise, is there an even greater risk of letting the inflation genie out of the bottle?

To conclude, our analysis suggests that the expansion of GVCs, facilitated by trade liberalisation and advances in technology, has put downward pressure on producer prices, with potential implications for monetary policy. Looking forward, if globalisation reverses, this may pose an upside risk to future inflation. In addition to the impact through competition that our work has focused on, increasing tariffs can exert more upward pressures on prices in the current context. This provides a further reason to resist the rising threat of trade protectionism in the global economy.

Lastly, if more intense competition in product and labour markets contributed to lower global inflation over recent decades, then it follows that waning structural reform ambition-against a backdrop of strengthening global growth-could lead to inflationary pressures. Given mounting evidence of increasing market power in certain sectors, policy efforts to adapt anti-trust and pro-competitive market 
regulations will not only bring benefits to long-run productivity growth, but will also be desirable from a monetary policy perspective.

${ }^{*}$ Dan Andrews is currently at the Australian Treasury and William Witheridge is at NeW YORK UNIVERSITY

COECD Observer No 314 Q2 2018

References

https://oe.cd/2sb https://oe.cd/2sb 